\title{
artigo
}

Mota, R. F. O., Leon, P. A. P., Oliveira, P. C. A., Xavier, T. G. M., Leal, N. P. R., Souza, L. F.

Avaliação da destreza manual de pessoas com esclerose múltipla usando o nine hole peg test

\section{Avaliação da destreza manual de pessoas com esclerose múltipla usando o nine hole peg test}

\author{
Assessment of manual dexterity in people with multiple sclerosis using the nine hole peg test \\ Evaluación de la destreza manual en personas con esclerosis múltiple mediante la prueba de clavija de nueve agujeros
}

\begin{abstract}
RESUMO
Objetivo: Avaliar a destreza manual de pessoas diagnosticadas com Esclerose Múltipla acompanhadas em um Centro de Referência. Método: Estudo de campo, exploratório, descritivo, com abordagem quantitativa, realizado em um público maior de 18 e menor de 60 anos, referente a 30\% de 186 indivíduos cadastrados, totalizando uma amostra de 55 pacientes, caracterizada como não probabilística por conveniência. Possuiu como variável o tempo de diagnóstico e a pontuação na EDSS, utilizando o nine hole peg test e um roteiro para a coleta em prontuários como instrumentos. Os dados foram estatisticamentre analisados de forma descritiva e analítica. Resultados: Pacientes com escore na EDSS < 3 apresentaram melhor desempenho no teste, enquanto pacientes com escore > 3 obtiveram dificuldades acentuadas, necessitando de maior tempo para executá-lo. Conclusão: Com base nos resultados, verifica-se que o escore na EDSS é diretamente proporcional ao desempenho da coordenação motora fina dos membros superiores.
\end{abstract}

DESCRITORES: Esclerose Múltipla; Neuroplasticidade; Destreza Motora; Sistema Nervoso.

\section{ABSTRACT}

Objective: To evaluate the manual dexterity of people diagnosed with Multiple Sclerosis followed up at a Reference Center. Method: Field study, exploratory, descriptive, with a quantitative approach, carried out in an audience over 18 and under 60 years old, referring to $30 \%$ of 186 registered individuals, totaling a sample of 55 patients, characterized as non-probabilistic for convenience. It had as a variable the time of diagnosis and the score in the EDSS, using the nine hole peg test and a script for the collection in medical records as instruments. The data were statistically analyzed in a descriptive and analytical way. Results: Patients with an EDSS score $<3$ performed better on the test, while patients with a score $>3$ had marked difficulties, requiring more time to perform it. Conclusion: Based on the results, it appears that the score on the EDSS is directly proportional to the performance of fine motor coordination of the upper limbs.

DESCRIPTORS: Multiple Sclerosis; Neuroplasticity; Motor Dexterity; Nervous system.

\section{RESUMEN}

Objetivo: Evaluar la destreza manual de personas con Esclerosis Múltiple seguidas en el Centro de Referencia. Método: Estudio de campo, exploratorio, descriptivo, con enfoque cuantitativo, realizado en una audiencia mayor de 18 y menor de 60 años, referido al 30\% de 186 inscritos, totalizando una muestra de 55 pacientes, caracterizados como no probabilísticos por conveniencia. . Tuvo como variable el tiempo de diagnóstico y la puntuación en la EDSS, utilizando como instrumentos la prueba de clavija de nueve hoyos y un guión para la recogida en historias clínicas. Los datos fueron analizados estadísticamente de forma descriptiva y analítica. Resultados: Los pacientes con una puntuación EDSS <3 se desempeñaron mejor en la prueba, mientras que los pacientes con una puntuación> 3 tuvieron marcadas dificultades, requiriendo más tiempo para realizarla. Conclusión: Con base en los resultados, parece que la puntuación en la EDSS es directamente proporcional al desempeño de la coordinación motora fina de los miembros superiores.

DESCRIPTORES: Esclerosis Múltiple; Neuroplasticidad; Motor de destreza; Sistema nervioso.

RECEBIDO EM: 24/03/2021 APROVADO EM: 08/04/2021

Rejane Ferreira de Oliveira Mota.

Graduação em Enfermagem. Unipê.

ORCID: 0000-0001-5777-4849.

Pollyana Amorim Ponce de Leon.

Graduação em Enfermagem. Mestre em Enfermagem pela UFPB. 
ORCID: 0000-0003-4291-9536.

Patrícia da Cruz Araruna Oliveira.

Graduação em Enfermagem. Doutora em Saúde Pública.

ORCID:0000-0003-4090-1228.

Thaís Grilo Moreira Xavier.

Graduação em Enfermagem. Mestre em Enfermagem pela UFPB.

ORCID: 0000-0002-1132-6482.

Natália Pessoa da Rocha Leal.

Graduação em Enfermagem. Mestre em Enfermagem pela UFPB.

ORCID: 0000-0001-6404-5580.

Luciana Ferreira de Souza.

Graduada em Enfermagem pela Universidade Federal da Paraíba - UFPB. Professora do Unipê. Mestra em Modelos de Decisão e Saúde pela UFPB.

ORCID: 0000-0002-5998-1216.

\section{INTRODUÇÃO}

A Esclerose Múltipla (EM) é uma doença crônico-degenerativa, não transmissível, autoimune, desmielinizante e inflamatória, que atinge o Sistema Nervoso Central (SNC), provocando uma série de incapacidades na funcionalidade de indivíduos, em sua maioria, na fase adulta, entre 18 a 55 anos de idade ${ }^{(1)}$. Um levantamento global, no ano de 2013, realizado pela Brazilian Committee for Treatment and Research in Multiple Sclerosis (BCTRIMS), mapeou 2,3 milhões de pessoas em todas as regióes do globo diagnosticadas com Esclerose Múltipla. Os estudos comprovaram o aumento de $10 \%$ de diagnósticos durante o intervalo de tempo referente ao quinquênio 20082013, junto ao aumento do número de aparelhos de ressonância magnética em países emergentes no mesmo período ${ }^{(2)}$.

Dados do Ministério da Saúde afirmam que a prevalência no Brasil é de 15 casos a cada 100.000 habitantes, totalizando 35.000 brasileiros dentre o índice mundial. O Sul do Brasil possui o maior índice de prevalência, totalizando 26/100.000 habitantes, seguido das regiões Sudeste apresentando 17-18/100.000 e Centro-Oeste com $10-13 / 100.000$. O Nordeste e o Norte possuem as menores taxas, com 8-10/100.000 e 4-5/100.000, respectivamente ${ }^{(3)}$. Por atingir indivíduos jovens requerendo destes
Um levantamento

global, no ano de

2013, realizado pela

Brazilian Committee

for Treatment and

Research in Multi-

ple Sclerosis (BC-

TRIMS), mapeou 2,3

milhões de pessoas

em todas as regióes

do globo diagnosti-

cadas com Esclerose

Múltipla uma série de mudanças e adaptações, vê-se que o impacto psicológico do diagnóstico junto a todo o processo clínico da doença traz medo e ansiedade, principais características da fase inicial de $\mathrm{EM}^{(4)}$.

Atrelado a esses fatores, verifica-se a deterioração motora e cognitiva que, ao longo do tempo, interferirá na qualidade de vida. Estima-se que $40-60 \%$ das pessoas com diagnóstico de EM possuam um declínio cognitivo levando a impactos no âmbito social, principalmente no meio profissional e/ou acadêmico dos pacientes, sendo estes, na maioria das vezes, jovens, no auge de suas produções ${ }^{(1)}$.

A memória de curto prazo, concentração, fala, compreensão de palavras, são sinais que podem indicar o início ou agravamento do déficit cognitivo. Já os sintomas de perda de força motora, disfunção neuromuscular, atrofia e perda de sensibilidade nos membros são sinais que podem indicar início ou agravo de déficit motor. As constataçôes desses tipos de deterioração não são comumente observadas, apesar de afetar e interferir no desenvolvimento profissional, comunicativo e nas relações/ atividades diárias, pois dificilmente o paciente, amigos, familiares e equipe médica interliga esses sinais e sintomas à EM. Relaciona-se a disfunção cognitiva e/ou motora mediante o número de lesões no encéfalo que se evidencia através de testes ou escalas específicas para este fim ${ }^{(5)}$. 
A neuroplasticidade caracteriza-se como a capacidade do cérebro lesionado de adequar-se e moldar-se a algum estímulo, agindo em dada área afetada ou parte responsável por realizar determinada ação que tenha sido prejudicada diante das lesões. A estimulação ocorre a partir de testes específicos que, em sua maioria, são internacionais, mas validados no Brasil, com orientação clara e objetiva para o paciente e profissional ${ }^{(6,7,8)}$.

Este estudo possibilitou a avaliação da performance dos membros superiores em uma atividade que mensuarava a destreza manual de indivíduos diagnosticados com EM, assistidos em um Centro de Referência em Esclerose Múltipla. Diante disso, surgiu o seguinte questionamento: as alterações de destreza manual são influenciadas pelo estado de incapacidade mensurado através da Expanded Disability Status Scale (EDSS)? Ou existem outras variáveis clínicas que estão associadas ao desempenho dos membros superiores?

\section{MÉTODO}

Trata-se de um estudo transversal, de campo, exploratório, descritivo, com abordagem quantitativa, realizado no município de João Pessoa-PB, no Centro de Referência em Esclerose Múltipla da Paraíba (CREMPB), nos meses de Fevereiro a Maio de 2019. A população alvo do estudo foi formada por pacientes diagnosticados com esclerose múltipla, atendidos no CREMPB e que se enquadravam nos critérios de inclusão.

Incluíram-se no estudo pacientes que fossem residentes do Município de João Pessoa e /ou regiões metropolitanas, que tinham mais de 18 e menos de 60 anos de idade, que fossem acompanhados pela equipe do CREMPB, tivessem a escolaridade mínima correspondente a 8 anos de estudo, tivessem sido avaliados quanto ao estado de incapacidade nos últimos 12 meses através da Expanded Disability Status Scale (EDSS), possuindo nessa escala, score que varie entre 0,0 a 6,0 e ter decidido participar do processo avaliativo, por livre e espontânea vontade, assinando o termo de
A neuroplasticida-

de caracteriza-se

como a capacidade

do cérebro lesiona-

do de adequar-se e

moldar-se a algum

estímulo, agindo em

dada área afetada ou

parte responsável por

realizar determinada

ação que tenha sido

prejudicada diante

das lesões. compromisso livre e esclarecido (TCLE).

Foram excluídos da coleta dos dados pacientes cadeirantes e aqueles que apresentaram algum evento de caráter físico ou emocional que os impossibilitaram de continuar a colaboração com sua participação. Da mesma forma, aqueles que, no instante da coleta, necessitaram se ausentar por motivos pessoais interrompendo o processo. Além disso, não fizeram parte da pesquisa os usuários que apresentaram, no momento da pesquisa, as manifestações clínicas neurológicas (surto).

A amostra equivaleu-se a $30 \%$ de um total de 186 indivíduos cadastrados, totalizando uma amostra de 55 pacientes, caracterizada como não probabilística por conveniência. As variáveis foram tempo de diagnóstico e pontuação na EDSS. Utilizou-se como instrumentos de coleta de dados o Nine Hole Peg Test (NHPT) e um roteiro sequenciado com tópicos a serem preenchidos com informações oriundas dos prontuários. A realização da coleta de dados foi composta por duas etapas, sendo a primeira constituída pelos dados demográficos, clínicos e epidemiológicos, extraídos dos prontuários, através do roteiro para essa coleta. Na segunda etapa foi aplicado o teste de destreza manual, integrantes de um instrumento internacional validado no Brasil, para os indivíduos diagnosticados com EM.

A bateria de testes "Multiple Sclerosis Functional Composite" (MSFC) é um instrumento de avaliação quantitativo, dividido em três partes, utilizado para estudos e ensaios clínicos em Esclerose Múltipla, classificado como padrão ouro. Foi desenvolvido com a finalidade de atingir três critérios, sendo estes, o multidimensionamento para refletir as várias expressões clínicas presentes na EM entre os pacientes, no decorrer do tempo, buscando a comprovação de mudanças que ocorrem de forma independente, ao longo do tempo. Esta, utiliza a medida da função psicomotora como componente obrigatório. Para tanto, os três componentes da MSFC medem as funções cognitivas e motoras, averiguando a deambulação, a funcionalidade dos membros superiores e a capacidade de cálculo e 
cognição (NMSS, 2019).

Da MSFC optou-se por aplicar o Nine Hole Peg Test (NHPT), que é um teste quantitativo que avaliou a função dos MMSS, onde ambas as mãos foram avaliadas duas vezes. $\mathrm{O}$ paciente sentou e teve na sua mesa um recipiente raso com nove pinos e um bloco de papelão contendo nove buracos vazios. No comando inicial o cronômetro foi acionado e o paciente pegou os nove pinos, um de cada vez, e os encaixou nos buracos o mais rápido que pôde, depois os removeu, um por vez, o mais rapidamente possível.

Foi realizado duas vezes por cada mão e o tempo total da tarefa foi registrado. O tempo de aplicação variou, mas maximizou-se em até 10 minutos. A pontuação foi dada através do tempo total das duas mãos juntas, convertido para a média das duas mãos juntas, finalizado com a média de cada mão separadamente e depois calculados. $\mathrm{O}$ tempo de administração do teste variou devido à particularidade clínica da pessoa com EM, no entanto, o tempo total de administração do teste foi entre 5 e 10 minutos. Quanto à sua administração, foi realizada pessoalmente por um examinador devidamente treinado, não se fazendo necessário ter sido um profissional médico ou enfermeiro. Finalizada a aplicação, foi gerada uma pontuação que pôde ser individual ou de forma composta.

$\mathrm{O}$ teste foi realizado em lugar privativo para que as informações coletadas se mantivessem sigilosas e, dessa forma, sido preservada a privacidade dos pacientes. No momento do teste esteve apenas o usuário do serviço, um acompanhante, caso solicitado, e o examinador que aplicou os testes. Da mesma forma, para que não fosse afetado o funcionamento do serviço, a aplicação do teste, bem como a coleta de dados nos prontuários, ocorreu em um espaço específico para tais ações. Todos os procedimentos para a coleta de dados foram antecedidos de planejamento entre a equipe do CREMPB, a partir de contato prévio da pesquisadora ao local da coleta, para que a preservação da rotina do serviço fosse mantida, e tais procedimentos, melhores ajustados à presença dos usuários no momento da pesquisa.

Após a etapa da coleta de dados, a análise foi realizada em dois grupos distintos, tendo sido o primeiro grupo constituído de indivíduos com score na EDSS de 0,0 a 3,0 e o segundo grupo por indivíduos com score na EDSS de 3,5 a 6,0. A tabulação desses dados aconteceu com o auxílio do programa Excel na versão 2016. Para a estatística descritiva e analítica foi utilizado o Statistical Package for the Social Sciences (SPSS) na versão 22.0. Utilizou-se o Teste $\mathrm{T}$ de Student, respeitando-se um nível de confiança de $95 \%$ e p-valor de 0,05 .

O Termo de Consentimento Livre e Esclarecido (TCLE) foi entregue impresso, antes da realização dos testes, para o participante assinar, autorizando então o uso das informações colhidas para a pesquisa. A mesma, foi aprovada com o CAAE $n^{\circ}$ 02804818.8.0000.5176.

\section{RESULTADOS}

Para verificar o desempenho médio dos grupos 1 (EDSS com escore $<3$, incapacidades mínimas) e grupo 2 (EDSS com escore $>3$, incapacidade entre mínima e moderada), utilizou-se o Teste T de Student que analisou os dados e gerou duas hipóteses de resultados: $\mathrm{H} 0=$ Que as médias de desempenho do Nine Hole Peg Test (NHPT) do grupo 1 e grupo 2 são iguais, ou, $\mathrm{H} 1$ = Que as médias de desempenho do Nine Hole Peg Test (NHPT) do grupo 1 são diferentes do grupo 2 .

A média de Tempo de Diagnóstico (TD) foi de 2,73 anos e a média do escore EDSS foi de 1,42, com mediana de 3,00
QUADRO 1- Medidas de frequência das

variáveis Tempo de Diagnóstico e EDSS, João Pessoa, Paraiba, Brasil, 2019, ( $n=55)$ :

$\begin{array}{lcc}\begin{array}{lcc}\text { ESTATÍSTICA } \\ \text { DESCRITIVA }\end{array} & \begin{array}{c}\text { TEMPO DE } \\ \text { DIAGNÓSTICO }\end{array} & \text { EDSS } \\ \begin{array}{l}\text { Estatística } \\ \text { Descritiva }\end{array} & 55 & 55 \\ \text { N } & 2,73 & 1,42 \\ \text { Média } & 3,00 & 1,00 \\ \text { Mediana } & 2 & 1 \\ \text { Moda } & 0,849 & 0,629 \\ \text { Desvio } & 0,721 & 0,396 \\ \text { Padrão } & 1 & 1 \\ \text { Variância } & 4 & 3 \\ \text { Mínimo } & & 1,00 \\ \text { Máximo } & 2,00 & 2,00 \\ \text { 10 Percentis } & & \\ 90 & 4,00 & \\ & & \end{array}$

para o Tempo de Diagnóstico e 1,00 para EDSS, sendo essa última a medida de frequência, que caracteriza o centro de distribuição da amostra.

A análise dos dados referente aos casos de EM por tempo de diagnóstico em anos, demonstra que há uma prevalência de pacientes com diagnóstico entre 1 a 5 anos, representando $41,8 \%$, sendo esta, a maior parte percentual dos resultados. Secundariamente, predomina os casos de EM com tempo de diagnóstico de 5 a 10 anos, referindo-se a 32,7\%.

Os EDSS's mais predominantes na EM foram os menores, integrados no primeiro descritor, que correspondem a EDSS's <

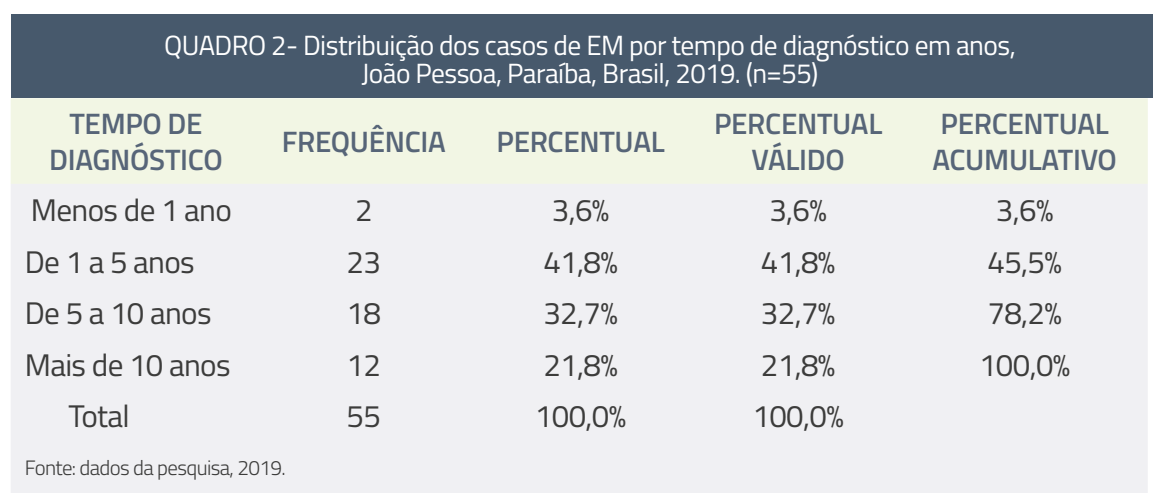




\section{artigo}

Mota, R. F. O., Leon, P. A. P., Oliveira, P. C. A., Xavier, T. G. M., Leal, N. P. R., Souza, L. F.

Avaliação da destreza manual de pessoas com esclerose múltipla usando o nine hole peg test

\begin{tabular}{lcccc}
\multicolumn{5}{c}{$\begin{array}{c}\text { QUADRO 3- Distribuição dos casos de EM de acordo com o EDSS } \\
\text { João Pessoa, Paraiba, Brasil, 2019. }(n=55):\end{array}$} \\
\hline EDSS & FREQUÊNCIA & PERCENTUAL & $\begin{array}{c}\text { PERCENTUAL } \\
\text { VÁLIDO }\end{array}$ & $\begin{array}{c}\text { PERCENTUAL } \\
\text { ACUMULATIVO }\end{array}$ \\
EDSS $<3$ & 36 & $65,5 \%$ & $65,5 \%$ & $65,5 \%$ \\
EDSS 3 $>5$ & 15 & $27,3 \%$ & $27,3 \%$ & $92,7 \%$ \\
EDSS 5 $>6$ & 4 & $7,3 \%$ & $7,3 \%$ & $100,0 \%$ \\
Total & 55 & $100,0 \%$ & $100,0 \%$ & \\
Fonte: dados da pesquisa, 2019. & & & &
\end{tabular}

\begin{tabular}{|c|c|c|c|c|c|}
\hline $\begin{array}{l}\text { ESTATÍSTICA DOS } \\
\text { GRUPOS }\end{array}$ & GRUPOS & $\mathrm{N}$ & MÉDIAS & $\begin{array}{l}\text { DESVIO } \\
\text { PADRÃOO }\end{array}$ & $\begin{array}{l}\text { MÉDIA } \\
\text { DE ERRO }\end{array}$ \\
\hline $\begin{array}{l}\text { Nine Hole Peg } \\
\text { Test }\end{array}$ & EDSS $<3$ & 36 & 21,0833 & 3,52579 & 0,58763 \\
\hline Mão Dominante & EDSS $>3$ & 19 & 27,5768 & 7,40422 & 1,69864 \\
\hline
\end{tabular}

reduzidos, causa no organismo feminino, se comparado ao masculino ${ }^{(2,3,4)}$. Estudos em torno da temática estão sendo desenvolvidos a fim de detectar quais fatores, com exatidão, influenciam ou determinam o surgimento da EM no sexo feminino, e quais fatores contribuem para o sexo masculino não progredir de igual modo com a doença $^{(5,6,7)}$.

O segundo fator sociodemográfico que foi analisado foi a faixa etária dos pacientes. Esta análise evidenciou a frequência dos casos de EM por idade, onde têm-se o maior quantitativo em 35 e 40 anos, com 45 anos como sequente. A média de idade para acometimento da EM é entre 18 a 55 anos de idade, havendo ocorrência também em menores e maiores que essa faixa etária ${ }^{(4)}$. Essa faixa é mais comumente acometida devido a elevadas doses de hormônios da puberdade, característica da fase juvenil,

\section{QUADRO 5- Estatística do Teste T de Student, João Pessoa, Paraíba, Brasil, 2019. (n=55):} TESTE T DE STUDENT- AMOSTRAS
INDEPENDENTE

Nine Hole

Peg Test

Variances assumed

Mão Do- Variances not

minante assumed

$16,83 \quad 0,000$

LEVENE'S TEST FOR EQUALITY VARIANCES

Fonte: dados da pesquisa, 2019.

\section{T STUDENT FOR EQUALITY OF MEANS}

95\% Confidence Interval of the Difference

$\begin{array}{ccc}\begin{array}{c}\text { Sig. } \\ \text { (2-tai- } \\ \text { led) }\end{array} & \begin{array}{c}\text { Mean Dif- } \\ \text { ference }\end{array} & \begin{array}{c}\text { Std. Error } \\ \text { Difference }\end{array} \\ 0,000 & -6,49351 & 1,46876 \\ 0,002 & -6,49351 & 1,79742\end{array}$

Lower

$-9,43946$

$-10,2172$
Upper

$-3,5476$

$-2,7698$
3, totalizando $65,5 \%$ do total da amostra. Segue o EDSS 3 a 5 com $27,3 \%$ e, como os menos predominantes, os pacientes com EDSS de 5 a 6, com registro de 7,3\%.

Os pacientes com EDSS $<3$ registraram melhor desempenho do membro superior dominante durante o Nine Hole Peg Test em relação ao tempo gasto para realização do teste, enquanto os pacientes que possuem EDSS > 3, obtiveram maior tempo para desenvolver a tarefa que os que possuem EDSS < 3 .

$\mathrm{O}$ Teste $\mathrm{T}$ de Student evidenciou em 95\% de confiabilidade que as médias de desempenho do Nine Hole Peg Test (NHPT) dos grupos 1 e 2 são diferentes, com Sig. < 0,05, com 53 graus de liberdade, portanto, rejeita-se $\mathrm{H} 0$. Assim, compreende-se que quanto maior o escore do EDSS, maior será o impacto no aspecto cognitivo de pessoas com diagnóstico de EM, especialmente sobre a coordenação motora fina dos MMSS.

\section{DISCUSSÃO}

O presente estudo, em sua abordagem sociodemográfica e cultural, aborda a princípio o acometimento da EM nos pacientes avaliados em relação à distribuição dos casos da doença por sexo, onde o feminino representa a parte majoritária com 76,36\% em relação a $23,64 \%$ do sexo masculino. Conforme o percentual demonstra, o sexo feminino é mais afetado pela Esclerose Múltipla que o masculino. Isso se dá devido a diversos fatores, sendo os principais os imunológicos, hormonais, genéticos, e o maior impacto que a Vitamina $\mathrm{D}$, em níveis permeiando-se pela idade adulta devido a fatores como gravidez, doenças psicossomáticas e afins, e estende-se à fase pré-senil, decorrentes das mudanças no organismo adulto, com influência da presença de DCNT's ${ }^{(4,5,6,7)}$.

Do mesmo modo, analisou-se a distribuição dos casos de EM ativos no mercado de trabalho por sexo e escolaridade destes pacientes, gerando como resultado a predominância do nível superior no público feminino, enquanto no masculino, predomina o nível médio. No entanto, o público masculino é mais ativo no mercado de trabalho que o feminino, segundo a análise dos dados. Sendo assim, é importante analisar o contexto ao qual a pessoa com EM está inserida, de modo a analisar seus ambientes e potenciais para o desenvolvi- 
mento psicomotor.Nesse aspecto, o quadro 1, que trata das medidas de frequência das variáveis Tempo de Diagnóstico e EDSS, traz que a média de Tempo de Diagnóstico (TD) foi de 2,73 anos e a média do escore EDSS foi de 1,42, com mediana de 3,00 para o Tempo de Diagnóstico e 1,00 para EDSS, sendo essa última a medida de frequência, que caracteriza o centro de distribuição da amostra.

A descrição da distribuição dos casos de EM por tempo de diagnóstico em anos que consta no quadro 2 , menciona que a maioria dos pacientes analisados possuem entre 1 e 5 anos de diagnó/stico, com $41,8 \%$, seguido de 5 a 10 anos com o segundo maior percentual de pacientes diagnosticados, com $32,7 \%$, tendo como consequentes, respectivamente, maiores que 10 anos, com representação percentual de $21,8 \%$ e menores que 1 ano com 3,6\%. O quadro 3 traz explícito os casos de EM de acordo com o EDSS. Verificou-se que $65,5 \%$ dos pacientes possuem EDSS menor que 3, assim como $27,3 \%$ possui EDSS entre 3 e 5 e, por último, 7,3\% dos pacientes que possuem o escore na EDSS maior que 5 e menor que 6. Optou-se por analisar até o escore 6 de um total de 10 , devido à incapacidade presente nos escores $>6$ em realizar as atividades necessárias para esta pesquisa.

Para avaliar a cognição, utilizou-se o Nine Hole Peg Test, sendo este, o padrão ouro para menurar a cognição e, mais especificamente, a coordenação motora dos MMSS dominante e não dominante. Após análise desses dados, conforme demonstra o quadro 4, contatou-se que os pacientes com
EDSS $<3$ obtiveram maior agilidade durante a realização dos testes e, consequentemente, foram mais rápidos e tiveram um melhor desempenho que os que possuíam escore na EDSS de 3 a 6. Notou-se que a coordenação motora ficou prejudicada com a doença e que, quanto maior o escore na EDSS, maior o comprometimento motor do paciente.

Feys et al ${ }^{(5)}$ relata que ao usar o Nine Hole Peg Test (NHPT), em uma amostra de 255 participantes, encontrou resultados semelhantes aos encontrados no presente estudo, em que pacientes com escore $>3$ na EDSS obtiveram rendimento menor em relação aos pacientes com escore $<3$ na respectiva escala. Do mesmo modo, ${ }^{(2)}$, em seu estudo com 105 participantes, evidenciou que a amostra, em sua totalidade, refletiu diminuição sensorial, desde os pacientes com EDSS leve $(<4)$ até os pacientes com EDSS grave não-ambulante (6- 6,5). Tal disfunção não aumentou com o EDSS mais elevado, porém, conforme maior o EDSS, menor o desenvolvimento da atividade do Nine Hole Peg Test (NHPT). Todos apresentaram destreza diminuída e, em sua maioria, apresentaram distúrbios bilaterais de sensibilidade, força e destreza, conforme evidenciou-se nesse estudo.

Por fim, o quadro 5 demonstra que o Teste T Student processou os dados e evidenciou a pesquisa como favorável ao que propôs alcançar. Para tanto, a Estatística do Teste T Student evidenciou em 95\% de confiabilidade que o Nine Hole Peg Test define que o desempenho dos pacientes foi avaliado através deste e que sua significância foi relevante para definir o quão afetada a função motora foi decorrente da EM e seu impacto no desempenho dos MMSS refletida na realização do NHPT.

\section{CONCLUSÃO}

Os resultados encontrados no presente estudo confirmaram que há comprometimento cognitivo nos pacientes com Esclerose Múltipla, e que quanto maior o escore na Expanded Disability Status Scale (EDSS), menor a desenvoltura cognitiva para realização de atividades que exijam maior uso da mesma. O que chama atenção nos resultados é a atuação da neuroplasticidade em readaptar-se à doença, de modo que o membro superior não dominante, atualmente, exerce as funções do dominante que foi afetado pela doença, de forma ideal tanto quanto a mão dominante.

Assim, compreende-se que a ocorrência da Esclerose Múltipla afeta a qualidade de vida dos pacientes, mais especificamente, sua função motora e cognitiva. Diante disso, faz-se necessária a atuação integral da equipe multidisciplinar, a fim de assistir o paciente em seu aspecto bio-psico-socio-cultural e espiritual, prestando uma assistência pautada em evidências científicas. Para tanto, torna-se imprescindível a realização de pesquisas e estudos que investiguem os aspectos evidenciados nesta pesquisa, bem como a avaliação da forma clínica e medicamentosa, aprofundando assim a temática.

\section{REFERÊNCIAS}

1. Brasil. Ministério da Saúde. Protocolo Clínico e Diretrizes Terapêuticas da Esclerose Múltipla. Fev. 2019. [cited 25 mai 2018]. Available from: http://conitec.gov.br/images/ consultas/2019/relator

io_PCDT_Esclerose_Multipla_CP03_2019.pdf.

2. Bertoni R, et al. Unilateral and bilateral upper limb dysfunction at body functions, activity and participation levels in people with multiple sclerosis. Mult Scler, v. 21, n. 12, p. 1566-1574, Fev. 2015. [cited 18 jun 2018]. Available from: https://www.ncbi.nlm.nih.gov/ pubmed/25662346.

3. Balsimelli, SF et al. Cognitive performance in patients with multiple sclerosis: Follow up to five years. Med Reabil, v. 30, n. 1, p. 3-6, Fev. 2011. [cited 15 jul 2018]. Available from: http://files.

bvs.br/upload/S/0103-5894/2011/v30n1/a2130.pdf

4. Bctrims. 0 que é Esclerose Múltipla? 2016. [cited 04 ago 2018]. Available from: https:// www.bctrims.org.br/esclerose-multipla/.

5. Feys et al. The Nine-Hole Peg Test as a manual dexterity performance measure for multiple sclerosis. Multiple Sclerosis Outcome Assessments Consortium. v. 23, n. 5, p. 711-720. 2017. [cited 30 ago 2018]. Available from: https:/www.ncbi.nlm.nih.gov/pmc/ articles/PMC5405844/.

6. Filippo et al. Neuroplasticidade e recuperação funcional na reabilitação pós-acidente vascular encefálico. Acta Fisiátr. v. 22, n. 2, p. 93-96. 2015. [cited 25 mai 2018]. Available from: http://www.actafisiatri

ca.org.br/detalhe_artigo.asp?id=585.

7. Silva, VF. et al. Brain stimulation used as biofeedback in neuronal activation of the temporal lobe area in autistic children. Arq. Neuro-Psiquiatr. São Paulo, v. 74, n. 8, p. 632-637, Aug. 2016. [cited 05 set 2018]. Available from: http://www.scielo.br/scielo.php?script=sci_arttext\&pid $=\mathrm{S} 0004282 \times 2016000800632 \& 1 \mathrm{ng}=e e \& n r m=i s s o$.

8. Ton, AMM, Vasconcelos, CCF, Alvarenga, RMP. Benign multiple sclerosis: aspects of cognition and neuroimaging. Arq. Neuro-Psiquiatr. São Paulo, v.75, n.6, p.394-401, June 2017. [cited 15 out 2018]. Available from: http://www.scielo.br/scielo.php?script=sci_arttext\&pid=S0004-282X2017000600394\&lng=en\&nrm=isso. 